\title{
GRIP: Generic Representatives in PRISM
}

\author{
Alastair F. Donaldson \\ Codeplay Software Ltd. \\ Edinburgh \\ ally@codeplay.com
}

\author{
Alice Miller \\ Department of Computing Science \\ University of Glasgow \\ alice@dcs.gla.ac.uk
}

\author{
David Parker \\ Computing Laboratory \\ Oxford University \\ david.parker@comlab.ox.ac.uk
}

\begin{abstract}
We give an overview of GRIP, a symmetry reduction tool for the probabilistic model checker PRISM, together with experimental results for a selection of example specifications.
\end{abstract}

\section{An Overview of GRIP}

GRIP (generic representatives in PRISM), introduced in [1], is a symmetry reduction tool for the PRISM model checker [6]. GRIP is based on the generic representatives approach of [2], which aims to overcome the inherent problem of combining symmetry reduction with symbolic state-space representation. We present an overview of GRIP version 2.0 (referred to henceforth as GRIP), an improved version of the original tool, and compare GRIP to PRISM-symm, an alternative symmetry reduction tool for PRISM [5]. GRIP, together with the PRISM examples used for experiments in Section 3 can be downloaded from our website [4].

The top panel of Figure 1 shows a simple leader election protocol in PRISM, adapted from [1]. The underlying model here is a Markov decision process (MDP). GRIP works by translating this specification into a reduced form, as shown in the bottom-left panel of the figure. The reduced specification abstracts away from specific modules, instead using a single generic module comprised of variables which count the number of modules in each potential local state. Symmetric temporal properties can also be translated into reduced form. PRISM can then be used, unchanged, to check reduced properties of a reduced specification.

\section{New Features of GRIP}

The original version of GRIP required specifications to consist of multiple instantiations of a single symmetric module type, specified using a single local state variable. This model of computation is in keeping with the presentation of the generic representatives approach for non-probabilistic model checking [2]. While a wide class of symmetric systems can, in theory, be specified in this way, accurately modelling complex protocols via a single state variable quickly becomes impractical.
GRIP now supports: multiple local state variables; a wide range of arithmetic and boolean expressions over these variables; communication via shared global variables, and multiple asymmetric modules in parallel with a single family of symmetric modules. In addition, GRIP handles models with continuous time Markov chain (CTMC) semantics.

Multiple local variables can result in a large number of local states, which translates to many counters in the specification output by GRIP. This in turn can lead to large MTBDDs (the symbolic data structure used by PRISM). To combat this, we have implemented an optimisation suggested in [3]: we use PRISM for local reachability analysis during the translation process, to reduce the number of counters in the output specification. In addition, since the sum of counter variables should always equal $N$ (the number of symmetric modules), the last counter variable can be eliminated and replaced with the formula $C_{k}=N-\left(\sum_{i=1}^{k-1} C_{i}\right)$. This second optimisation offers a modest reduction in MTBDD size. The bottom-right panel of Figure 1 shows the effect of these optimisations: local reachability analysis determines that the local state $(0,1)$ (where init $i=0$ and reg $i=1$ ) is unreachable, eliminating the need for the no_l variable and associated statements. The no_3 variable is then replaced with a formula.

\section{Experimental Results and Discussion}

Figure 2 summarises experimental results for model building with PRISM, PRISM-symm and GRIP (with and without optimisations) on five case studies, all of which are described in detail at [6]. The consensus, byzantine and rabin models are MDPs; $f g f$ and peer2peer are CTMCs. For reasons of space we have omitted model checking times, but note that all symmetry-reduced models are feasible to model check. In all cases, translation into reduced form using GRIP took less than two seconds. Experiments were performed on a $2.80 \mathrm{GHz}$ PC with $1 \mathrm{~GB}$ RAM.

It is not surprising that exploiting symmetry leads to a large state-space reduction. However, for symbolic model checking it is the MTBDD size for the resulting symmetric model that determines whether the technique is feasible. 


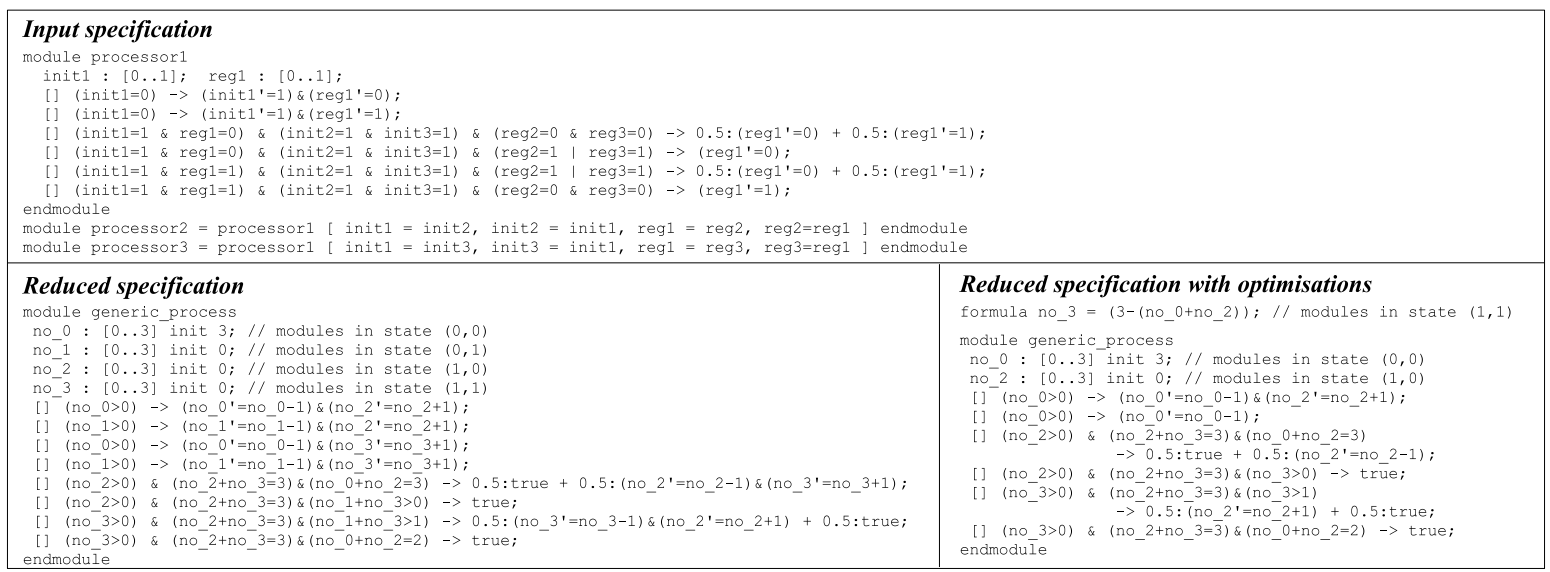

Figure 1. Applying GRIP to a simple leader election specification, with and without optimisations.

\begin{tabular}{|c|c|c|c|c|c|c|c|c|c|c|c|}
\hline \multirow[t]{2}{*}{ Case study } & \multirow[t]{2}{*}{$\mathrm{N}$} & \multicolumn{2}{|c|}{ States } & \multicolumn{4}{|c|}{ MTBDD (nodes) } & \multicolumn{4}{|c|}{ Build time (sec.) } \\
\hline & & Full & Symm & PRISM & $\begin{array}{l}\text { PRISM } \\
\text {-symm }\end{array}$ & GRIP & $\begin{array}{l}\text { GRIP } \\
\text { (opt.) }\end{array}$ & PRISM & $\begin{array}{l}\text { PRISM } \\
\text {-symm }\end{array}$ & GRIP & $\begin{array}{l}\text { GRIP } \\
\text { (opt.) }\end{array}$ \\
\hline consensus: Aspnes \& & 12 & $1.2 \mathrm{e}+11$ & 339,729 & 50,037 & 50,741 & 56,374 & 36,819 & 5.65 & 7.85 & 7.21 & 6.95 \\
\hline Herlihy's randomised & 14 & $5.0 \mathrm{e}+12$ & 747,243 & 78,171 & 79,123 & 78,072 & 51,192 & 9.44 & 14.5 & 11.41 & 10.2 \\
\hline consensus protocol & 16 & $2.1 \mathrm{e}+14$ & $1,497,972$ & 115,385 & 116,691 & 123,743 & 80,485 & 19.1 & 26.8 & 22.43 & 17.9 \\
\hline byzantine: randomised & 8 & $6.4 \mathrm{e}+8$ & 298,993 & 713,143 & 167,587 & 175,046 & 167,372 & 20.8 & 23.40 & 16.3 & 17.1 \\
\hline Byzantine agreement & 12 & $1.0 \mathrm{e}+13$ & $7,994,813$ & $4,257,996$ & 937,484 & 681,580 & 646,455 & 144.1 & 169.7 & 37.1 & 37.2 \\
\hline protocol & 16 & $1.9 \mathrm{e}+16$ & $1.1 \mathrm{e}+8$ & $13,306,326$ & $2,949,979$ & $1,986,234$ & $1,874,953$ & 975.5 & 1143 & 157.6 & 160.1 \\
\hline rabin: Rabin's & 6 & $1.3 \mathrm{e}+8$ & 356592 & 206,213 & 408,291 & mem-out & 185,943 & 7.56 & 16.9 & - & 46.7 \\
\hline randomised mutual & 7 & $2.5 \mathrm{e}+9$ & 1271328 & 287,661 & 587,917 & mem-out & 261,474 & 12.5 & 28.7 & - & 50.9 \\
\hline exclusion algorithm & 8 & $4.5 \mathrm{e}+10$ & 4062048 & 381,184 & 796,324 & mem-out & 430,901 & 20.2 & 46.2 & - & 102.7 \\
\hline$f g f:$ simplified version & 6 & $9.6 \mathrm{e}+7$ & 283,360 & 522,063 & $1,044,350$ & $1,784,685$ & $1,222,992$ & 47.4 & 75.2 & 73.2 & 46.4 \\
\hline of the FGF signalling & 8 & $4.1 \mathrm{e}+10$ & $3,996,135$ & $2,080,931$ & $4,114,456$ & $7,344,006$ & $5,119,910$ & 323.4 & 497.2 & 420.8 & 272.7 \\
\hline pathway & 10 & $1.7 \mathrm{e}+13$ & $4.0 \mathrm{e}+7$ & $6,314,340$ & $12,024,036$ & $18,660,241$ & $13,264,807$ & 2,135 & 3,028 & 2,047 & 1,275 \\
\hline peer 2 peer: simple & 5 & $3.4 \mathrm{e}+7$ & 376,992 & 26,266 & 101,630 & 157,476 & 157,476 & 0.133 & 1.32 & 2.79 & 4.30 \\
\hline $\mathrm{P} 2 \mathrm{P}$ protocol based & 6 & $1.1 \mathrm{e}+9$ & $2,324,784$ & 40,591 & 189,704 & 247,122 & 247,122 & 0.269 & 2.99 & 3.80 & 5.29 \\
\hline on BitTorrent & 7 & $3.4 \mathrm{e}+10$ & $1.3 e+7$ & 54,916 & 306,123 & 355,721 & 355,721 & 0.516 & 5.64 & 4.63 & 6.14 \\
\hline
\end{tabular}

Figure 2. Experimental results using PRISM, PRISM-symm, GRIP and optimised GRIP.

In this respect, GRIP's optimisations are clearly effective: optimised GRIP outperforms PRISM-symm on MTBDD size for all MDP examples and is comparable for the larger CTMC examples. GRIP also offers an improvement in building time for larger $f g f$ models. Note that although both GRIP and PRISM-symm produce larger MTBDDs than PRISM for the CTMC models, the reduction in state spaces make much larger models now amenable to model checking. On the rabin examples, GRIP requires longer to build models than the other techniques. This is due to complex expressions which arise in the translated PRISM code.

Despite our improvements to GRIP, PRISM-symm can be applied to a wider variety of examples where modules communicate via synchronisation labels. This restriction means that GRIP cannot handle certain case studies, such as a CSMA protocol, on which PRISM-symm performs well [5]. Another distinction between GRIP and PRISM-symm is that PRISM-symm tends to out-perform GRIP when applied to a specification consisting of a relatively small number of complex modules, whereas GRIP wins out when applied to a large number of simpler modules.

On the other hand, an important advantage of GRIP is that, unlike PRISM-symm, there is no need to first construct the full unreduced model. Further, since GRIP merely acts as a pre-processor for PRISM specifications, it automatically provides symmetry reduction for tools which use the PRISM input language, e.g. Ymer, or an input language into which PRISM specifications can be translated, e.g. MRMC.

To improve performance, we are considering techniques to further reduce MTBDD size and reduce the complexity of GRIP's program output.

\section{References}

[1] A. F. Donaldson and A. Miller. Symmetry reduction for probabilistic model checking using generic representatives. In ATVA'06, LNCS 4218, pp. 9-23. Springer, 2006.

[2] E. A. Emerson and T. Wahl. On combining symmetry reduction and symbolic representation for efficient model checking. In CHARME'03, LNCS 2860, pp. 216-230. Springer, 2003.

[3] E. A. Emerson and T. Wahl. Efficient reduction techniques for systems with many components. ENTCS 130:379-399.

[4] GRIP website: www.prismmodelchecker.org/grip

[5] M. Kwiatkowska, G. Norman, and D. Parker. Symmetry reduction for probabilistic model checking. In CAV'06, LNCS 4144, pp. 234-248. Springer, 2006.

[6] PRISM website: www.prismmodelchecker.org 